\title{
Survey on Load Balancing and Scheduling Algorithms in Cloud Integrated Fog Environment
}

\author{
Jyoti Bisht $^{1}$ and Dr. V V Subrahmanyam ${ }^{2}$ \\ ${ }^{1}$ Research Scholar, School of Computer and Information Sciences, IGNOU, New Delhi, INDIA \\ \{Email: Jyotibisht8891@gmail.com\} \\ ${ }^{2}$ Director, School of Computer and Information Sciences, IGNOU, New Delhi, INDIA \\ \{Email: vvsubrahmanyam@ignou.ac.in\}
}

\begin{abstract}
As technologies like IoT are emerging, increasing number of users on internet and workload on Cloud is increasing at an enormous rate along with the requirements of network bandwidth. Also the increasing demand for real time analytics and real time applications lead to the requirement of a new technology. Fog Computing in collaboration with cloud computing can provide a solution for all such requirements. This technology brings cloud computing resources like memory, computation near end users and henceprovides a solution.End devices like gateways, routers, cell base stations can be used as fog devices. Since these devices have limited resources, soon they will get overloaded.Hence proper scheduling and load balancing mechanism is needed to fully utilize the benefits of this technology. Various algorithms proposed to resolve the issue of task scheduling, resource provisioning and load balancing are discussed in this paper. We have also identified some of their shortcomings for further development.
\end{abstract}

Keywords- Fog computing, Cloud Computing, Scheduling, Load balancing.

\section{Introduction}

In the age of information technology, data is major commodity, and way of possessing and managing this data tends to generates more datadriven businesses value. Cisco estimated that 50 billion devices will be connected by 2020[1] with the increasinguse of Internet of Things (IoT) and hence will lead to massive amount of data generation.It also estimated in its white paper that data center IP traffic will reach 20.6 Zettabytes per year by the end of 2021 from 6.8 ZB per year in 2016 as is given in Fig 1. It is also estimated that social networking (26\% CAGR from 2016 to 2021) and video streaming (24 percent CAGR from 2016 to 2021) will be the fastest growing applications over internet. Also, the total amount of data created by any device will reach 847 ZB per year by 2021 from 218 ZB per year in 2016 due to emerging popularity of Internet of Things [2]. Another paper by Cisco Visual Networking Index (VNI) estimated overall IP traffic to grow from 122 EB per month in 2017 to 396 EB per month by 2022[3] as given in Fig 2. 


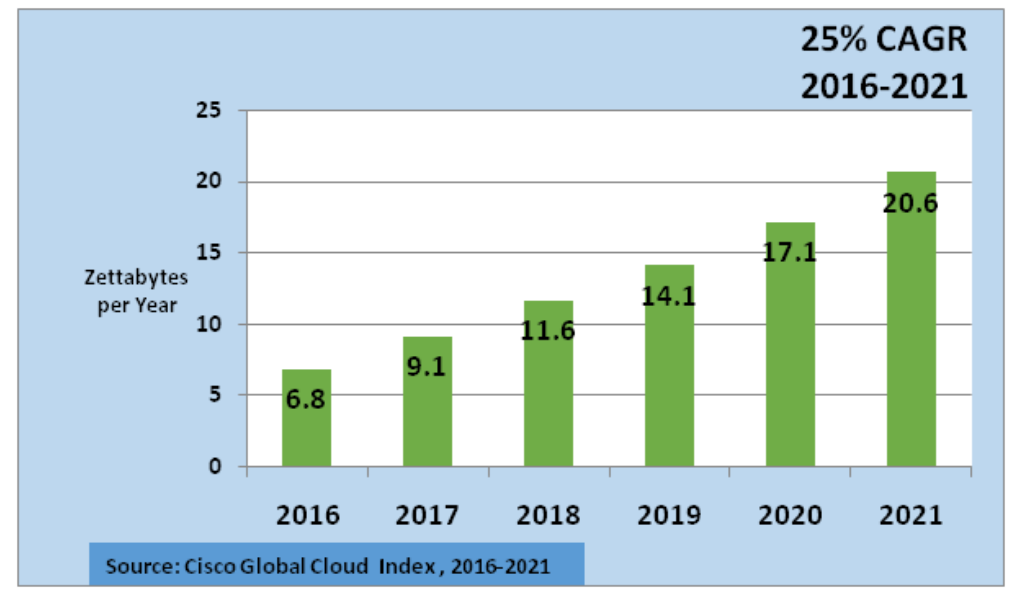

Fig. 1. Global data center IP traffic growth

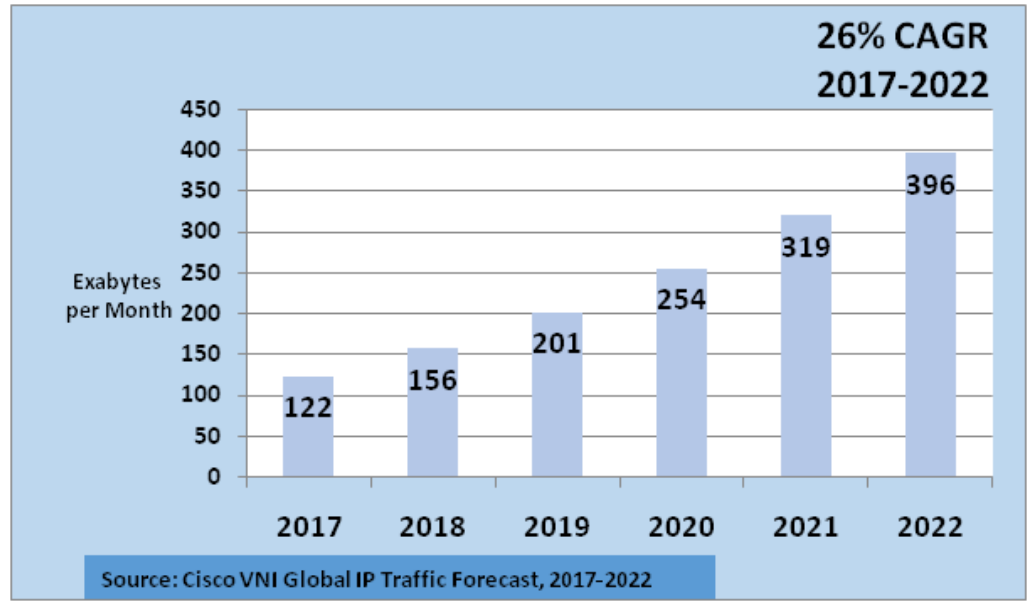

Fig. 2. Overall IP traffic expected to grow

Cloud offers a solution by delivering reliable services to its users by the use of data centers based on virtualization technology in which a single machine can act as multiple virtual machines and hence can handle requests from multiple users at a time independently. Consumers from anywhere in the world can access services provided by cloud on demand. Instead of consumers owning resources and managing themselves, current pay per use Cloud computing technology is an efficient solution since customers don't have to pay for initial setup cost and also not have to bother about the management, networking, licensing, technical issues and others. However, this technology is not suitable for latency-sensitive applications like monitoring of patients, real-time applications, traffic management, self-driving cars, cognitive assistance, since it require computing nodes to be present nearby to fulfil the request within some time constraints. It is difficult for cloud computing technology to satisfy 
requirements of low latency, mobility, location awareness and other applications that demands real time responses, since cloud servers are centralized and remotely located.

It would be infeasible and inefficient to use cloud in many applications due to bandwidth constraints since volume and velocity of data, which are two identified characteristics of Bigdata is increasing day by day. Also it would not be appropriate solution for many applications like email, photo storing, online medical records, and others due to privacy concerns.

\subsection{Fog Computing and its Architecture}

In January 2014, Cisco given a term Fog Computing to a technology that extends computing to the edge of the network. It is a way to bring cloud computing capabilities to the edge of the network.Fog computing can act as a middle layer between the cloud and IoT devices. It can provideservices of computing, storage, networking, and data management within the close vicinity of IoT devices and users on the network. It can be called as an extension of cloud computing. Benefits provided by cloud can still be provided with these extensions to fog, which includes, manageability, containerization, virtualization, orchestration, and others but with better efficiency.

OpenFog Consortium or at present known as Industrial Internet Consortiumdefined fog computing as "a horizontal system level architecture that distributes computing, storage, control and networking functions closer to the users along a cloud-to-thing continuum" [4].

Traditional cloud computing model can continue to remain as a centralized computing systems having major responsibilities with few capabilities shifted towards or offered by fog devices as a distributed system. The selection or decision of which tasks should go to fog layer and which tasks should be forwarded to the backend cloud are specific to applications and depends upon the resource requirements and availability of resources and time constraints. These decisions could be taken statically or can change dynamically depending upon network state changes in areas like load onavailable storage, processor, bandwidth, fault events, security, and cost.

A three-layer generalized logical architecture for fog computing is depicted in Fig 3. The three layers are IoT or end devices at end layer, Fog servers at fog layer as middle layer, and Cloud servers at the cloud layer. 


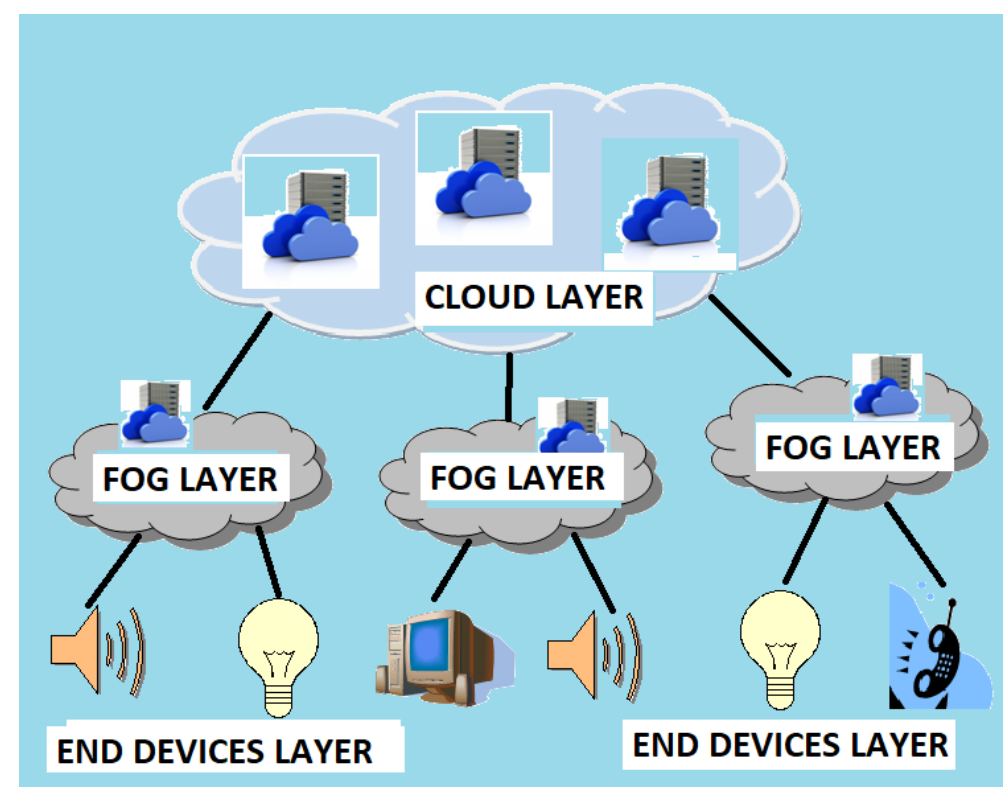

Fig 3: Fog Computing Architecture

A non proprietary fog computing architecture called Open Fog architecture is also established to support existing cloud computing services in addition to diverse IoT and edge-oriented ecosystem and identifiedscalability, agility, security, openness, among other "pillars" of open fog architecture [4].IEEE Standards Association later adopted Open Fog Consortium's reference architecture as a standard for fog computing through IEEE 1934[6].

The various characteristic of fog computing identified are edge location, location awareness,geographical distribution, low latency, large-scale sensor networks, mobility support, real-time computations, heterogeneity, wireless access, interoperability and federation[7] and its application scenarios are Smart Grid, Smart Traffic Lights, Wireless Sensor and Actuator Networks and Connected Vehicles, Decentralized Smart Building Control [5,8-9].

However, several obstacles are there which are yet unsolved research challenges andare needed to be solved to get full benefit from this emerging technology. Research challenges identified are - general purpose computing on fog nodes, discovering edge nodes, task placement, application placement, partitioning of tasks and offloading, improving quality-ofservice $(\mathrm{QoS})$ and experience $(\mathrm{QoE})$, security and privacy issues $[8,10]$.

\section{Load Balancing and Scheduling in Fog computing}

As discussed above, Fog computing architecture has three layers- end devices, middleware fog layer and centralized cloud servers at cloud layer. A fog layer in fog computing architecture 
consists of several fog servers, where each fog server can contains multiple virtual machines. Also, each fog server like a cloud server must have processing power, communication unit and data storing capabilities.

Users can communicate directly with fog servers using wireless interface like Bluetooth, WIFietc since they are present in the vicinity of users. Therefore,fog servers independent of the cloudservers, should provide pre-defined applications and pre-cached information to benefitsusers. Also, fog servers are connected to cloud servers by using wired or wireless connections so that users can have access to more services and resources available at cloud since limited resources are available at Fog servers.

Fog servers like cloud servers are based on virtualization technology. Multiple users request can be served by multiple virtual machines created in fog servers. If larger request arrives at fog layer,the request can be decomposed into a set of tasks, which canbe then assigned to a several virtual machines. Decision of allocation of task to which virtual machine is taken by a scheduling technique decided by fog server. The aim of job or task scheduling technique is to optimally assign various tasks to virtual machines at fog layer with minimum use of resources like shortest execution time, least memory use. The job scheduling problem is NP-hard problem since there is no deterministic polynomial algorithm to solve it.

Also, multiple tasks can be submitted to a fog node from multiple users. It is also possible that large number of requests can arrive at aparticular server forcing it to become overloaded soon in a situation like peak hours, while other fog servers are idle or lightly loaded. Hence proper balancing of load between servers and placement decision of servers areimportant for successful implementation of Fog Computing.

\subsection{Categorization of Load Distributing Algorithms}

Load distribution can improve the performance of fog computing by transferring tasks from fog server which are overloaded or service quality is reducing due to over burdening, to servers which are available to serve more requests or can prove better quality of service (QoS) due to availability of more or free resources. By this tasks can utilize resourcesthat would other- wise go waste. This can lead to better performance and efficient utilization of resources.Load-distribution algorithms can be implemented in three ways- dynamic, static and adaptive.

Static load distribution algorithms do not make use of system's state information i.e. load on system.Based on previous knowledge about the system, decisions are taken. These algorithms can hence lead to poor decisions making because they do not consider node's state when making such decisions. It is also possible that a task which initially arrived at an idle node istransferred to a node which is already heavily loaded.

Dynamic load distribution algorithms use system's state information to make decisions. These algorithms must collect previous information, store it, analyze it and also make use of current load on the system. Use of state information canimprove quality of decisions hence dynamic algorithms have the ability to perform better then static algorithms.But maintaining state information and analysing that data can causes overhead to the system. 
Adaptive load-distribution algorithms are a special type of dynamic algorithms. According to the changing system state, they can dynamically adapt new policies and change their parameters i.e. with the change in the scenarios or situations, algorithms known to perform better in that situation are implemented and when the scenario changes, different algorithm can be used to fulfil the needs.

The decision of load balancing and scheduling can be made centrally i.e., by a single designated device or distributed i.e., by all the devices in a system. Decision taken by a single system can save time and resources but can easily become bottleneck for the entire system, whereas decision taken by group of nodes may take better decision but at the cost of coordination time.

Various algorithms for load balancing and scheduling are available in cloud computing technology [11] which after some modifications can be applied to fog computing environment since as compared to cloud, resources are limited, devices are heterogeneous in nature and tasks are latency sensitive.

\section{Exploratory Study on Load Balancing and Scheduling Algorithms}

Researchers addressed the issues of scheduling, load balancing, task allocation or resource allocation in the fog integrated cloud environment in many research papers given below and most of them addressed the centralized scheduling, load balancing and offloading of tasks. In centralized version of the problem, either entire information about the tasks, network, or nodes is known previously, or a centralized system decides task should be allocated to which device.In thedistributedversionoftheproblemthereisnocentral device responsible for allocation of tasks and not evenfullinformationaboutthetasks, devices or network isknown.

Ningning et al. [12]proposed a task allocation techniquebased on graph partitioning in fog computing environment. Here, depending upon the level of resources required, tasks can be assigned to a single or multiple virtual machines. Physical nodes of the fog computing layer are represented here as a non-directional graph. A minimum spanning tree is constructed from the graph obtained and edges that do not provide enough resources are removed. The tree obtained hence represents partition that can be used for task allocation. It is demonstrated that it leads to improvement in tasks' run time. Limitation of this approach is that frequent graph repartitioning is required when there is change in fog devices.

SalimBitama et al. [13] proposed Bees Life Algorithm (BLA) which is a bio-inspired optimizing approach for job scheduling problem in the fog computing environment. An architecture in proposed in which user when sends a request to a nearby fog, it forwards data and parameters of that request to administrator node. The administrator node will decompose the job into a set of tasks and executes BLA to find the node which best executes the tasks and forwards the task to the corresponding node. Administratornode after getting partial results from the devices prepares final and forwards it to the user. It is demonstrated that the algorithm requires less memory and lesser execution time than PSO (particle swarm optimization) and genetic algorithm. 
TejaswiniChoudhari et al. [14] proposed priority based task scheduling algorithm in the fog integrated cloud architecture. The algorithm implemented first checks the availability of desired resources in fog servers to satisfy user's requirement and if resources are not available, the request is forwarded to the cloud layer. Here three queues of different priority level maintained- high, medium and low.According to the maximum allowed delay and the priority given by user, priority is given to a task.It is shown that priority levels when added toOptimize Response Time (ORT) and Reconfigure Dynamically (RD) algorithm in cloud only environment reduces cost but increases response time. It is also tested that when priority is added to efficient resource allocation (ERA) algorithm in fog cloud integrated environment, it reduces both response time and cost. However major drawback in this paper is that it considers static priority levels and does not consider classical problem of aging with priorities.

LindongLiu et al. [15] used Classification Mining technique forscheduling of task. I-Apriori algorithm is introduced after applying some modification on Apriori algorithm and using it association rules are generated. Rules generated are input to TSFC algorithm (Task Scheduling in Fog Computing)which schedules tasks to execute on fog devices so as to minimize the completion time. It is shown that TSFC gives reduced execution and waiting time than MCT, MET, and MIN-MIN but it does not considered bandwidth between processors.

JinlaiXu et al. [16] proposed Zenith, a model for allocation of resources. This model can be used for establishing contracts between service providers and infrastructure providersfor edge layer, for allocation of resources so as to maximize profit for both and to reduce latency for user. Allocation of resources here is decided by using auction mechanism in which firstly, utility function for both the service providers and infrastructure providers is identified,and thenstrategy for bidding is given. Finally an algorithm is introduced to decide selection of winner. Performance of this model when compared with Coupled edge computing (CEC) and conventional cloud model gives better response time for any number of servers in edge layer and better utilization and success rate when number of server increases.

Changlong Li et al. [17] proposed SSLB (self-similarity-based load balancing)technique, for fog servers spread over large scale by using both centralized and decentralized mechanisms. In this, k-means clustering algorithm is used to group fog nodes into cells. Scheduling is done independently in each cell. Workload migration acrosscells is done based on two adaptive threshold policy called Task Distributing and Task Grasping. It is further shown that utilization of resources in SSLB is better than DS (based on Karger's work) and more stable than CS (based on Apache Hadoop). It is also shown that with increasing number of fog nodes SSLB performs better than centralized scheme and better than decentralized scheme with reduced network bandwidth.

Xuan-Qui Pham and et al. [18] proposed a distributed task scheduling algorithm CMaS (CostMakespan aware Scheduling) in which fog computing works in collaboration with cloud computing.Its objective is minimize both execution time of application and the cost ofresources. It consists of three phases-task prioritizing based on upward ranking, node selection phase to assign task to a fog or cloud node based on utility function, and task reassignment phase to meet user defined deadlines. It is also shown that CMaS performs better 
thanGfC(greedy for cost) in terms of performance and better than HEFT and CCSH in terms of cost.

Guangshun Liet al. [19] proposed a resource scheduling algorithm called FCAP which uses FCM ( fuzzy C-Means clustering) to find cluster of fog resources and PSO (particle swarm optimization) to obtain global optimization which reduces scale of resource search and then weightedmatchingmethodisusedtomatch theuserrequest and the scheduling scheme is retuned. It is shown that FCAP converges faster than FCM algorithm. It is also shown that in comparison with the Min-min algorithm, this algorithm can lead to better user satisfaction.

SabiheKabirzadeh et al. [20] proposed hyper-heuristic algorithm for scheduling in fog environment. Testing and selection technique is used to identify best algorithm for the new workflow among the available algorithms. In training phase, for initial set of workflows, different heuristic algorithms GA,PSO,ACO,and SA are implemented to allocate resources. After calculation of energy consumed, total cost and usage of network, it is stored in a database. In the test phase, for each new input topology, a row of training dataset having least Euclidean distance with input is selected and algorithm best for that topology is chosen (mentioned in database table). It is further shown that it consumes less energy than other heuristic algorithms and average cost is improved.

SagarVerma et al. [21] proposed load balancing algorithm based on replication technique for a fog integrated cloud environment. By maintainingreplicated data in fog networks, dependency on clouddata centers will reduce leading to reduced latency time. The request from the client when reaches the nearest fog server and if it is not capable of providing the data and resources itself the request is forwarded to the adjacent server in fog tier for handling the request. If the fog tier server fails to provide data or services requested by the user, the request is migrated to the cloud tier. Cloud server will then process the request if the request data is available there. If the cloud server also cannot serve the request due to un-availability of data or resources, it broadcasts the query packet throughout the Cloud tier. It is shown thatthis algorithm gives reduced response time than Round Robin and Throttledbut high cost due to data transfer.

Fatima et al. [22] proposed dynamic service broker policy (DSP) in fog integrated cloud environment for energy management inresidential buildings in all regions of the world. Depending upon the allocation of VMs, the fog device which is able to manage load with minimum delay is selected, otherwise request is forwarded to cloud.It is shown that the DSP policy when comparison to dynamic configure with load (DR) policy for RR algorithm, gives better overall response time and processing time but the cost of virtual machines increases.

Maria et al. [23] proposed integration of cloud with smart grid for proper utilization of energy by users. Here performance maximizationis mathematically formulatedand performance of round robin (RR), throttled and weighted round robin (WRR) algorithms is analysed with various service broker policies under cloud fog environment. It is shown that Throttled algorithm performs better than RR and WRR.

SamanZahoor et al. [24] used PSO algorithm in cloud-fog environment which can give globally best solution in addition to locally best. For each iteration, current local best value or global best value of previous iteration, whichever meets the fitness criteria, gets selected for 
current iteration. Simulations are done to show that PSO minimizes response time and cost as compared to RR and Throttled.

Ye Yu et al. [25] proposedSDLB (scalable and dynamic load balancer) for fog and MEC. Here POG data structureis used to develop SDLB, which makes use of minimal perfect hashing technique. It is also shown that SDLB when compared with other load balancer design making use of hash table and consistent hashing is faster and consumes less memory.

Huynh et al [26]proposed TCaS algorithm based on genetic algorithm to allocate bag of tasks in cloud integrated fog environment to minimize both execution time and cost of operations.Here, a utility function is formulated to achieveminimize makespan and cost. Genetic algorithm is used with two-point crossover operation and Darwin's lawof survival is used as selection strategy. Simulations are done to show that TCaS performs better than BLA for task completion time and achieves better fitness value of cost and makespan trade-off.

Deng et al. [27] tried to achieve balance between transmission delay and power consumption in the fog integrated cloud environment.In this paper workload allocation problem is formulated in which minimizing the power consumption with fulfillingdelay constraint in is dividedinto three sub-problems- tradeoff between power consumption and delay in fog layer, cloud layer and minimization of communication delay for dispatching, which can be solved within corresponding sub-systems.Hungarian method is used to solve delay minimization sub problem. Simulations shows that with increase in workload, power consumed and processing delay increases for fog only systems, power increases but delay constant in cloud only system and power increases and delay decreases in fog integrated cloud system.

Lin $\mathrm{Gu}$ et al. [28] investigated association of user with a base station, distribution of tasks and placement of VM in order to minimize cost in medical cyber-physical systems. The problem is firstly formulated into anon-linear linear program of mixed-integer (MINLP), which is then linearized into mixed-integer linear programming (MILP). Heuristic algorithm based on twophase linear programmingis proposed to solve the sub problems.Results of this algorithm is compared with greedy algorithm to shows that 2 phase LP gives minimum incremental cost which includesboth VM deployment cost andcommunication cost between base stations.

Oueis et al [29] proposed a heuristic algorithm for resources managementfor clustering of fog servers which supports customizable objective like minimizing latency or minimizing power consumption, etc in the small cell cloud. In this paper, establishment of clusters and allocationof resources is split into two phases- First, computational resources are allocated locally to small cell. Secondly, clusters (SCC) are established for user left in first step due to scared resources. To reduce complexity of problem, optimal cluster is found for each user, independently of the others. Three algorithms proposed are- EDF-PC (Earliest Deadline First with minimizing power consumption), EDF-LAT (Earliest Deadline First with minimizing latency), and CS-LAT (computation size with minimizing latency). The result shows that EDF-LAT and CS-LAT gives better user satisfaction than static and no clustering. Also they achieved significant latency gain compared to others and EDF-PC consumes lesser power than other two proposed techniques. 
Xueying Guo et al. [30] proposed atask assignment policy in Edge-cloud system to reduce both power consumption and delay. Firstly the problem is modelled as a continuous-time queuing system andequivalent discrete-time Markov decision problem is proposed to make problem tractable. To reduce the complexity, an associated dual problem is analysed. Finally, an Indexing policy is proposed to handle complex problem and reduce overhead of communication. It is used to analyzing dual of the original problem in which index are calculated based on their queuing status based on which users forwards request to cloud or edge servers

Yousefpour et al.[31] proposed and formulated a cooperative offloading policy to forward request from IoT devices to neighbouring fog nodes (Fog-to-Fog) or to forward them to the cloud (IoT-Cloud or Fog-Cloud) for delay minimization. If estimated waiting time on a fog node is smaller than a threshold value, the task is acceptedotherwise offloaded. There is a limit on number of times a task can be forwarded to a neighbouring node, after which a task must be forwarded to cloud. It is shown through simulations that AFP (proposed policy-IoT process or forwards to cloud or fog) outperforms LFP (Light fog processing-only light request forwarded to fog or else to cloud if not processes by IoT) and NFP (no fog processing-IoT if not process can only forwards the tasks to cloud). AFP also gives lesser delay than Index policy [30].A table showing the comparative study of various algorithms is given below.

Table 1 Comparison of Different Scheduling and Load Balancing Algorithms

\begin{tabular}{|c|c|c|c|c|c|}
\hline $\begin{array}{l}\text { Author(s) } \\
\text { and Year }\end{array}$ & Main ideas & $\begin{array}{l}\text { Simulation } \\
\text { tool / } \\
\text { Programming } \\
\text { Language }\end{array}$ & $\begin{array}{l}\text { Network } \\
\text { type }\end{array}$ & Improved criteria & Limitation \\
\hline $\begin{array}{l}\text { Ningning et } \\
\text { al. } 2016[12]\end{array}$ & $\begin{array}{l}\text { Task allocation based } \\
\text { graph partitioning } \\
\text { Constructed Minimum } \\
\text { spanning tree }\end{array}$ & $\begin{array}{l}\text { Extended } \\
\text { Hadoop }\end{array}$ & Fog & Task's run time & $\begin{array}{l}\text { Frequent graph } \\
\text { repartitioning needed } \\
\text { for dynamic load }\end{array}$ \\
\hline $\begin{array}{l}\text { Salim et al. } \\
2017 \text { [13] }\end{array}$ & $\begin{array}{l}\text { Central node } \\
\text { decomposes jobs into } \\
\text { tasks. } \\
\text { Bee Life Algorithm } \\
\text { (BLA) is used to allocate } \\
\text { task to nodes }\end{array}$ & $\mathrm{C}++$ & Fog & $\begin{array}{l}\text { Reduced execution time } \\
\text { and memory than PSO } \\
\text { and genetic algorithm }\end{array}$ & $\begin{array}{l}\text { Administrator node is } \\
\text { central, hence may } \\
\text { become bottleneck or } \\
\text { single point of failure. } \\
\text { Small dataset used }\end{array}$ \\
\hline $\begin{array}{l}\text { Tejaswini et } \\
\text { al. } 2018 \text { [14] }\end{array}$ & $\begin{array}{l}\text { Priority assigned to task } \\
\text { based on given priority } \\
\text { and max delay allowed. }\end{array}$ & Cloud Analyst & Cloud -Fog & $\begin{array}{l}\text { Reduced the response } \\
\text { time and cost as } \\
\text { compared to ORT and } \\
\text { RD algorithm. }\end{array}$ & $\begin{array}{l}\text { Considers static } \\
\text { priority levels. } \\
\text { May suffer from } \\
\text { problem of aging. }\end{array}$ \\
\hline $\begin{array}{l}\text { LindongLiu } \\
\text { et al. } 2018 \\
{[15]}\end{array}$ & $\begin{array}{l}\text { Based on Classification } \\
\text { Mining. } \\
\text { Used I-Apriori (modified } \\
\text { Apriori) }\end{array}$ & SimGrid & Fog & $\begin{array}{l}\text { Reduced execution } \\
\text { andwaiting time than } \\
\text { MCT, MET, and MIN- } \\
\text { MIN algorithm. }\end{array}$ & $\begin{array}{l}\text { Bandwidth between } \\
\text { processors not } \\
\text { considered }\end{array}$ \\
\hline
\end{tabular}




\begin{tabular}{|c|c|c|c|c|c|}
\hline & $\begin{array}{l}\text { Introduced TSFC } \\
\text { algorithm }\end{array}$ & & & & \\
\hline $\begin{array}{l}\text { JinlaiXu et } \\
\text { al. } 2017 \text { [16] }\end{array}$ & $\begin{array}{l}\text { Based on an auction- } \\
\text { based mechanism for } \\
\text { resource allocation. } \\
\text { Decoupled service and } \\
\text { infrastructure } \\
\text { management. }\end{array}$ & $\begin{array}{l}\text { geographic } \\
\text { map }\end{array}$ & Edge & $\begin{array}{l}\text { Reduced response time } \\
\text { and } \\
\text { Increased utilization }\end{array}$ & $\begin{array}{l}\text { Low utilization with } \\
\text { scarce resources } \\
\text { Assumes every } \\
\text { container requires } \\
\text { equal resources }\end{array}$ \\
\hline $\begin{array}{l}\text { Changlong } \\
\text { Li et al. } \\
2018 \text { [17] }\end{array}$ & $\begin{array}{l}\text { Uses both centralized } \\
\text { and distributed technique } \\
\text { k-means clustering used } \\
\text { to group nodes. } \\
\text { To avoid communication } \\
\text { overhead,adaptive } \\
\text { threshold policy is used }\end{array}$ & iFogSim & Fog & $\begin{array}{l}\text { Resource utilization of } \\
\text { SSLB better than } \\
\text { traditional centralized } \\
\text { and decentralized } \\
\text { techniques. } \\
\text { Performs better for large } \\
\text { scale clusters. }\end{array}$ & $\begin{array}{l}\text { Overhead of clustering. } \\
\text { Network load due to } \\
\text { probing and } \\
\text { communication. }\end{array}$ \\
\hline $\begin{array}{l}\text { Xuan-Qui } \\
\text { Pham and } \\
\text { et al. } \\
2017[18]\end{array}$ & $\begin{array}{l}\text { Based on utility function } \\
\text { that considers both cost } \\
\text { of resources and } \\
\text { finishing time of task. } \\
\text { Performs task } \\
\text { reassignment for meeting } \\
\text { deadlines. }\end{array}$ & CloudSim & Cloud-Fog & $\begin{array}{l}\text { Achieves trade-off } \\
\text { between cost of } \\
\text { resources and } \\
\text { performance. } \\
\text { Reduced cost than HEFT } \\
\text { and CCSH } \\
\text { Improved performance } \\
\text { than GfC }\end{array}$ & $\begin{array}{l}\text { CMaS cannot } \\
\text { guarantee finishing of } \\
\text { tasks before deadline. } \\
\text { May not satisfy } \\
\text { deadline for GE } \\
\text { program }\end{array}$ \\
\hline $\begin{array}{l}\text { Guangshun } \\
\text { Li and et al. } \\
2019 \text { [19] }\end{array}$ & $\begin{array}{l}\text { Based on FCM and PSO } \\
\text { algorithm. } \\
\text { Creates cluster of fog } \\
\text { resources to reduce } \\
\text { search and uses weighted } \\
\text { matching method to } \\
\text { allocate resources. }\end{array}$ & MATLAB & Fog & $\begin{array}{l}\text { Better user satisfaction } \\
\text { than min-min algorithm } \\
\text { Faster convergence than } \\
\text { FCM algorithm }\end{array}$ & $\begin{array}{l}\text { One task can be } \\
\text { handled at a time } \\
\text { Does not consider } \\
\text { dynamic nature of } \\
\text { resources. }\end{array}$ \\
\hline $\begin{array}{l}\text { SabiheKabi } \\
\text { rzadeh et al. } \\
2017[20]\end{array}$ & $\begin{array}{l}\text { Hyper-heuristic } \\
\text { algorithm based on } \\
\text { classification technique. } \\
\text { Consists of training and } \\
\text { testing phases. }\end{array}$ & iFogSim & Fog & $\begin{array}{l}\text { Reduced energy } \\
\text { consumption and } \\
\text { simulation time than } \\
\text { other heuristic } \\
\text { algorithms } \\
\text { Improved decision } \\
\text { making according to } \\
\text { workflow }\end{array}$ & $\begin{array}{l}\text { Initial training phase is } \\
\text { an overhead and } \\
\text { involves delay is start- } \\
\text { up time. }\end{array}$ \\
\hline $\begin{array}{l}\text { SagarVerm } \\
\text { a et al. } \\
2016[21]\end{array}$ & $\begin{array}{l}\text { Uses data replication } \\
\text { technique. } \\
\text { Proposed Cloud-Fog } \\
\text { architecture. }\end{array}$ & Cloud Analyst & Cloud-Fog & $\begin{array}{l}\text { Reduceresponse time } \\
\text { than RR and Throttled. } \\
\text { Reduced processing time } \\
\text { on data center } \\
\text { Reduced dependency on } \\
\text { cloud layer }\end{array}$ & $\begin{array}{l}\text { Increases total cost due } \\
\text { to data transfer for } \\
\text { replication. }\end{array}$ \\
\hline
\end{tabular}




\begin{tabular}{|c|c|c|c|c|c|}
\hline $\begin{array}{l}\text { Fatima et } \\
\text { al. } 2018[22]\end{array}$ & $\begin{array}{l}\text { New service broker } \\
\text { technique introduced. }\end{array}$ & Cloud Analyst & Cloud-Fog & $\begin{array}{l}\text { reducedprocessing } \\
\text { response time }\end{array}$ & $\begin{array}{l}\text { cost of VMs increased } \\
\text { Considered virtual } \\
\text { machines with similar } \\
\text { capabilities }\end{array}$ \\
\hline $\begin{array}{l}\text { Maria } \\
\text { Naeem et al. } \\
2019[23]\end{array}$ & $\begin{array}{l}\text { Implemented Weighted } \\
\text { round robin algorithm in } \\
\text { fog-cloud environment. } \\
\text { Mathematically } \\
\text { formulated performance } \\
\text { objective. }\end{array}$ & Cloud Analyst & Cloud-Fog & $\begin{array}{l}\text { Considers different load } \\
\text { distribution for different } \\
\text { VM capabilities, hence } \\
\text { balances load }\end{array}$ & $\begin{array}{l}\text { Response time } \\
\text { increases as compared } \\
\text { to throttled algorithm. }\end{array}$ \\
\hline $\begin{array}{l}\text { SamanZaho } \\
\text { or et al. } \\
2018 \text { [24] }\end{array}$ & $\begin{array}{l}\text { Implemented PSO in } \\
\text { fog-cloud for resource } \\
\text { management in SG }\end{array}$ & Cloud Analyst & Cloud-Fog & $\begin{array}{l}\text { Reduces response time } \\
\text { and cost as compared to } \\
\text { RR and Throttled }\end{array}$ & $\begin{array}{l}\text { It is specific for two } \\
\text { buildings }\end{array}$ \\
\hline $\begin{array}{l}\text { Ye Yu et al. } \\
2017[25]\end{array}$ & $\begin{array}{l}\text { Proposed SLDB } \\
\text { (Scalable and Dynamic } \\
\text { Load Balancer). } \\
\text { POG data structure } \\
\text { (minimal perfect } \\
\text { hashing) }\end{array}$ & $\begin{array}{l}\text { HashMap } \\
\text { using a self- } \\
\text { balancing } \\
\text { binary search } \\
\text { tree }\end{array}$ & MEC-Fog & $\begin{array}{l}\text { Less memory utilization } \\
\text { Faster and better data } \\
\text { plane throughput }\end{array}$ & $\begin{array}{l}\text { Structures grows } \\
\text { linearly with number of } \\
\text { stateful IDs } \\
\text { Time to rebalance } \\
\text { increases with no. of } \\
\text { packets }\end{array}$ \\
\hline $\begin{array}{l}\text { Huynh et al. } \\
2018[26]\end{array}$ & $\begin{array}{l}\text { Utility function to } \\
\text { achieve trade-off } \\
\text { between costs and } \\
\text { makespan. } \\
\text { Genetic algorithm with } \\
\text { two-point crossover is } \\
\text { used. }\end{array}$ & iFogSim & Cloud-Fog & $\begin{array}{l}\text { Less time to complete } \\
\text { tasks than BLA } \\
\text { Better fitness value than } \\
\text { BLA }\end{array}$ & $\begin{array}{l}\text { Cost of TCaS is more } \\
\text { than BLA. } \\
\text { Tasks are assumed to } \\
\text { be independent. }\end{array}$ \\
\hline $\begin{array}{l}\text { Deng et al. } \\
2016[27]\end{array}$ & $\begin{array}{l}\text { Workload allocation is } \\
\text { divided into three sub- } \\
\text { problems } \\
\text { Hungarian method is } \\
\text { used }\end{array}$ & MATLAB & Cloud-Fog & $\begin{array}{l}\text { Power consumption and } \\
\text { delay }\end{array}$ & $\begin{array}{l}\text { Implemented in } \\
\text { centralized system }\end{array}$ \\
\hline $\begin{array}{l}\text { Lin Gu et } \\
\text { al. } 2015[28]\end{array}$ & $\begin{array}{l}\text { Developed two-phase } \\
\text { linear programmingbased } \\
\text { on heuristic }\end{array}$ & $\begin{array}{l}\text { Mathematical } \\
\text { Gurobi }\end{array}$ & Fog & $\begin{array}{l}\text { Reduced deployment and } \\
\text { communication cost }\end{array}$ & $\begin{array}{l}\text { Does not provide } \\
\text { computational } \\
\text { offloading capability. }\end{array}$ \\
\hline $\begin{array}{l}\text { Oueis et al. } \\
2015 \text { [29] }\end{array}$ & $\begin{array}{l}\text { Resource allocation and } \\
\text { cluster establishment } \\
\text { using EDF-PC, EDF- } \\
\text { LAT, CS-LAT. } \\
\text { Heuristic }\end{array}$ & $\begin{array}{l}\text { 3GPP } \\
\text { framework }\end{array}$ & Fog & $\begin{array}{l}\text { Improved user } \\
\text { satisfaction. } \\
\text { EDF-PC is power } \\
\text { efficient. } \\
\text { Customizable algorithm } \\
\text { for multi user scenario }\end{array}$ & $\begin{array}{l}\text { Complex for large } \\
\text { scale fog infrastructure }\end{array}$ \\
\hline $\begin{array}{l}\text { Xueying } \\
\text { Guo et al. } \\
2016[30]\end{array}$ & $\begin{array}{l}\text { Used Markov decision } \\
\text { problems for online task } \\
\text { allocation. } \\
\text { Index policy is proposed. }\end{array}$ & theoretical & Cloud-Fog & $\begin{array}{l}\text { Reduced power } \\
\text { consumption. } \\
\text { Reduced delay }\end{array}$ & $\begin{array}{l}\text { Continuous-time } \\
\text { queuing system }\end{array}$ \\
\hline
\end{tabular}




\begin{tabular}{|l|l|l|l|l|l|}
\hline $\begin{array}{l}\text { Yousefpour } \\
\text { et al. 2018 } \\
\text { [31] }\end{array}$ & $\begin{array}{l}\text { Task off-loading } \\
\text { collaboration scheme. } \\
\text { Offloading decisions } \\
\text { based on queuing status }\end{array}$ & $\begin{array}{l}\text { Not } \\
\text { Mentioned }\end{array}$ & Cloud-Fog & Minimization of delay & $\begin{array}{l}\text { Threshold parameter is } \\
\text { kept static }\end{array}$ \\
\hline
\end{tabular}

\section{Conclusion}

In this paper, we have surveyedfog integrated cloud environment and its 3-layered architecture.We have identified the need for the development of new algorithms for scheduling and load balancing for fog integrated cloud computing keeping in view to reducing the latency time.This paper also gives an exploratory comparative study of the existing algorithms proposed by various researchers and also set grounds for further improvements and development of these algorithms. Since selecting the best fog server (or edge server) in order to minimize latency for every end device and maximizing resource utilizationis NP-Hard problem, researchers are using various optimization techniques like graph theory, heuristics, local search, stochastic search, genetic algorithms, deep learning. Pros and cons of all the algorithms were discussed and the whole summary is placed in a tabular form. Asfog computingtechnology is in itsinfant stage, there is lot of scope for improvement of the existing algorithms or defining new ones.

\section{References}

[1]. DaveEvans. The internet of things: How the next evolution of the internet is changing everything. CISCO whitepaper, 2011.

[2]. Cisco Global Cloud Index. Forecast and Methodology, 2016-2021. CISCO whitepaper. 2018

[3]. Cisco Visual Networking Index. Forecast and Trends, 2017-2022 White Paper.Updated:February 27. 2019.

[4]. Open Fog Consortium. Open fog reference architecture for fog computing. Feb 2017. [Online]. Available: https://www.openfogconsortium.org/ra.

[5]. Subhadeep Sarkar, SudipMisra. Assessment of the Suitability of Fog Computing in the Context of Internet of Things.IEEE. 2015.

[6]. IEEE Standard Association. Ieee 1934-2018 - ieee standard for adoption of openfog reference architecture for fog computing. [Online]. Available: https://standards.ieee.org/standard/1934-2018.html [7]. F. Bonomi, R. Milito, J. Zhu, and S. Addepalli. Fog Computing and Its Role in the Internet of Things. Proceedings of ACM; MCC pp. 13-16. 2012.

[8]. Ivan Stojmenovic , Sheng Wen. The Fog Computing Paradigm: Scenarios and Security Issues. Proceedings of Federated Conference on Computer Science and Information Systems, 2014. IEEE ;2014.

[9]. F. Bonomi, R. Milito, J. Zhu, and S. Addepalli. Fog Computing and Its Role in the Internet of Things. Proceedings of ACM ; MCC pp. 13-16. 2012

[10]. Blesson Varghese, Nan Wang, SakilBarbhuiya. Challenges and Opportunities in Edge Computing. arXiv:1609.01967v1 [cs.DC] 7 Sep 2016.

[11].Subhadra Bose Shaw , Dr. A.K. Singh. A Survey on Scheduling and Load Balancing Techniques in Cloud Computing Environment. IEEE; 2014

[12]. SONG Ningning1, GONG Chao, AN Xingshuo, ZHAN Qiang. Fog Computing Dynamic Load balancing mechanism based on Graph Repartitioning. China Communications , March 2016.

[13]. SalimBitam, SheraliZeadally,AbdelhamidMellouk. Fog computing job scheduling optimization based on bees swarm. Published online: 7 Informa UK Limited,Taylor\& Francis Group. 10 Apr 2017. 
[14]. Tejaswini Choudhari, Melody Moh, Teng-Sheng Moh. Prioritized Task Scheduling in Fog Computing. USA, ACM ; 2018

[15]. LindongLiu, DeyuQi, NaqinZhou, andYilinWu. A Task Scheduling Algorithm Based on Classification Mining in Fog Computing Environment. Wireless Communications and Mobile Computing Volume, Wiley, Hindawi; 2018.

[16]. JinlaiXu, BalajiPalanisamy, Heiko Ludwig and Qingyang Wang. Zenith: Utility-aware Resource Allocation for Edge Computing. IEEE International conference on Edge computing ,2017.

[17].Changlong Li,Hang Zhuang,Qingfeng Wang,Xuehai Zhou. SSLB: Self-Similarity-Based Load Balancing for Large-Scale Fog Computing. Arabian Journal for Science and Engineering, December 2018, Volume 43, Issue 12, pp 7487-7498.

[18]. Xuan-Qui Pham, Nguyen Doan Man, Nguyen Dao Tan Tri, Ngo Quang Thai and Eui-Nam Huh. A cost- and performance-effective approach for task scheduling based on collaboration between cloud and fog computing. International Journal of Distributed Sensor Networks. 2017. Vol. 13(11).

[19]. Guangshun Li , Yuncui Liu, Junhua Wu , Dandan Lin andShuaishuai Zhao. Methods of Resource Scheduling Based on Optimized Fuzzy Clustering in Fog Computing. Sensors, MDPI;2019.

[20]. SabiheKabirzadeh, DadmehrRahbari, Mohsen Nickray. A Hyper Heuristic Algorithm for Scheduling of Fog Networks. proceeding of the 21st conference of fruct association, IEEE ;2017.

[21]. SagarVerma, Arun Kumar Yadav, Deepak Motwani. An Efficient Data Replication and Load Balancing Technique for Fog Computing Environment. Proceeding of 3rd International Conference on Computing for Sustainable Global Development, IEEE; Oct 2016.

[22]. Itrat Fatima, NadeemJavaid, Muhammad Nadeem Iqbal, IsraShafi, Ayesha Anjum, UbedUllah Memo. Integration of Cloud and Fog based Environment for Effective Resource Distribution in Smart Buildings.14th International Wireless Communications \& Mobile Computing Conference (IWCMC).IEEE; Aug 2018.

[23]. Maria Naeem, NadeemJavaid, MaheenZahid, Amna Abbas, Sadia Rasheed, and SaniahRehman. Cloud and Fog Based Smart Grid Environment for Efficient Energy Management. INCoS 2018, Springer 2019, LNDECT 23. pp. 514-525.

[24]. SamanZahoor, NadeemJavaid, Asif Khan, BibiRuqia Fatima J. Muhammad, Maida Zahid. A Cloud-Fog-Based Smart Grid Model for Efficient Resource Utilization. 14th International Wireless Communications \& Mobile Computing Conference (IWCMC). IEEE; August 2018.

[25]. Ye Yu, Xin Li, and Chen Qian. Sdlb.:A scalable and dynamic software load balancer for fog and mobile edge computing.Proceedings ofthe Workshop on Mobile Edge Communications. ACM 2017. pages 55-60.

[26]. Huynh ThiThanhBinh, Tran The Anh, Do Bao Son, Pham AnhDuc, Binh Minh Nguyen. An Evolutionary Algorithm for Solving Task SchedulingProblem in Cloud-Fog Computing Environment. International Symposium on Information and Communication Technology ,SoICT. ACM; 2018. [27].Ruilong Deng, Rongxing Lu, ChengzheLai,Tom H. Luan, andHaoLiang. Optimal Workload Allocation in Fog-Cloud Computing Toward Balanced Delay and Power Consumption. IEEE Internet of Things Journal. Dec 2016; Volume: 3 Issue: 6.

[28]. Lin Gu, Deze Zeng, Song Guo, Ahmed Barnawi, and Yong Xiang. Cost Efficient Resource Management in Fog Computing Supported Medical Cyber-Physical System. IEEE Transactions on Emerging Topics in Computing. December 2015; Volume: 5, Issue: 1,Jan.-March 2017.

[29]. Jessica Oueis, Emilio Calvanese Strinati, and Sergio Barbarossa. The Fog Balancing: Load Distribution for Small Cell Cloud Computing. IEEE 81st Vehicular Technology Conference (VTC Spring); 2015.

[30]. Xueying Guo, Rahul Singh, Tianchu Zhao and Zhisheng Niu. An Index Based Task Assignment Policy for Achieving Optimal Power-Delay Tradeoff in Edge Cloud Systems. IEEE International Conference on Communications (ICC); 2016.

[31]. Ashkan Yousefpour, Genya Ishigaki, Riti Gour, and Jason P. Jue. On Reducing IoT Service Delay via Fog Offloading. IEEE Internet of Things Journal. April 2018; Volume: 5 Issue: 2. 